\title{
Appendix
}

\section{Na'o sentences}

(52) Context:

$\mathrm{Na}$ alu Alana 'i le taulaga analeila 'ae nofo Asovale i le fale. 'O a mea a

Asovale na fai?

'Alana went to town earlier while Asovale stayed home. What did Asovale do?'

(53) Vna'oOA:

Na tapena na'o le fa'apusa e Asovale analeila.

PAST prepare only DET earth oven ERG Asovale earlier 'Asovale only prepared the earth oven earlier.'

(54) VAna'oO:

Na tapena e Asovale na'o le fa'apusa analeila.

PAST prepare ERG Asovale only DET earth oven earlier 'Asovale only prepared the earth oven earlier.'

(55) na'oOVA:

Na'o le fa'apusa na tapena e Asovale analeila. only DET earth oven PAST prepare ERG Asovale earlier 'It was only the earth oven that Asovale prepared earlier.'

(56) Context:

$\mathrm{Na}$ alu Alana 'i le taulaga analeila 'ae nofo Asovale i le fale. 'O ai na tapena na'o le umu analeila?

'Alana went to town earlier while Asovale stayed home. Who only prepared the earth oven?'

(57) AVna'oO:

'O Asovale na tapena na'o le fa'apusa analeila.

PRES Asovale PAST prepare-ES only DET earth oven earlier 'It was Asovale who only prepared the earth oven.'

(58) Context:

Na alu Alana 'i le taulaga analeila 'ae nofo Asovale i le fale. 'Aisea 'ua lelava ai Asovale?

'Alana went to town earlier while Asovale stayed home. Why is Asovale tired?'

(59) na'o AVO:

Na'o Asovale na tapena le fa'apusa analeila. only Asovale PAST prepare-ES

DET earth oven earlier 'It was only Asovale who prepared the earth oven earlier.'

(60) Context:

$\mathrm{Na}$ fai le fa'atau a Alana ina 'ua 'uma ona matamata i le tifaga ananafi. Na le mautonu po'o le a lana mea 'ole'a fa'atau. 'O le a le fa'atau a Alana na fai?

'Alana did some shopping after watching a movie in town yesterday. She was struggling to make up her mind on what to buy. What did she end up buying?'

(61) Vna'oOA:

$\mathrm{Na}$ filifili na'o le lavalava e Alana ananafi.

PAST choose only DET sarong ERG Alana yesterday

'Alana only chose a sarong yesterday.'

(62) VAna'oO:

$\mathrm{Na}$ filifili e Alana na'o le lavalava ananafi. 
PAST choose ERG Alana only DET sarong yesterday

'Alana only chose a sarong yesterday.' na'oOVA:

Na'o le lavalava na filifili e Alana ananafi. only DET sarong PAST choose ERG Alana yesterday

'It was only a sarong Alana chose yesterday.'

Context:

$\mathrm{Na}$ fai le fa'atau a Alana ina 'ua 'uma ona matamata i le tifaga ananafi.

Na le mautonu po'o le a lana mea 'ole'a fa'atau. 'O ai na filifilia na'o le lavalava?

'Alana did some shopping after watching a movie in town yesterday.She was struggling to make up her mind on what to buy. Who chose only a sarong?'

(65) AVna'oO:

'O Alana na filifilia na'ole lavalava ananafi.

PRES Alana PAST choose-ES only DET sarong yesterday

'It was Alana who chose only a sarong.'

(66) Context:

$\mathrm{Na}$ fai le fa'atau a Alana ina 'ua 'uma ona matamata i le tifaga ananafi. $\mathrm{Na}$

le mautonu po'o le a lana mea 'ole'a fa'atau. Aisea na foliga 'ese mai ai

Alana mai le isi 'uma vaega?

'Alana did some shopping after watching a movie in town yesterday.She was struggling to make up her mind on what to buy. Why did Alana look different from everyone else?'

(67) na'o $A V O$ :

Na'o Alana na filfilia le lavalava ananafi.

only Alana PAST choose-ES DET sarong yesterday

'It was only Alana who chose a sarong yesterday.'

(68) Context:

Na nofo Manino i le fale e fai ana fe'au ananafi. 'O a ana tiute na fa'atino?

'Manino stayed home to do some chores yesterday. What chores did he do?'

(69) (Vna'oOA:

$\mathrm{Na}$ fa'amama na'o le ma'umaga e Manino ananafi.

PAST clear only DET plantation ERG Manino yesterday 'Manino only cleared the plantation yesterday.'

(70) VAna'oO:

Na fa'amama e Manino na'o le ma'umaga ananafi.

PAST clear ERG Manino only DET plantation yesterday 'Manino only cleared the plantation yesterday.'

(71) na'oOVA:

Na'o le ma'umaga na fa'amama e Manino ananafi. only DET plantation PAST clear ERG Manino yesterday 'It was only the plantation that Manino cleared yesterday.' 
(72) Context:

Na nofo Manino i le fale e fai ana fe ' au ananafi. ' Aisea ' ua mitamita ai le tama o Manino ' ia te ia?

'Manino stayed home to do some chores yesterday. Why was Manino s father proud of him?

(73) AVna'oO:

'O Manino na fa' amamaina na' o le ma ' umaga ananafi.

PRES Manino PAST clear-ES only DET plantation yesterday 'It was Manino who

cleared only the plantation yesterday.

(74) Context:

Na nofo Manino i le fale e fai ana fe ' au ananafi.' O ai na fa' amamaina le ma umaga?

'Manino stayed home to do some chores yesterday. Who cleared the plantation?

(75) na'o AVO:

$\mathrm{Na}$ ' o Manino na fa' amamaina le ma' umaga ananafi. only Manino PAST

clear-ES DET plantation yesterday 'It was only Manino who cleared the plantation yesterday. 